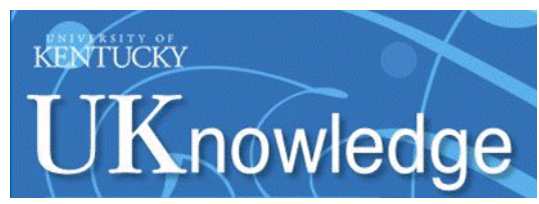

University of Kentucky

UKnowledge

\title{
Specificity of the Minimal Clinically Important Difference of the Quick Disabilities of the Arm Shoulder and Hand (QDASH) for Distal Upper Extremity Conditions
}

\author{
Enrique V. Smith-Forbes \\ Fort Sam Houston Clinic, e_vsf12@hotmail.com \\ Dana M. Howell \\ University of Kentucky \\ Jason Willoughby \\ Drayer Physical Therapy Institute \\ Donald G. Pitts \\ Drayer Physical Therapy Institute \\ Timothy L. Uhl \\ University of Kentucky, tluhl2@uky.edu \\ Follow this and additional works at: https://uknowledge.uky.edu/rehabsci_facpub \\ Part of the Rehabilitation and Therapy Commons
}

Right click to open a feedback form in a new tab to let us know how this document benefits you.

\section{Repository Citation}

Smith-Forbes, Enrique V.; Howell, Dana M.; Willoughby, Jason; Pitts, Donald G.; and Uhl, Timothy L., "Specificity of the Minimal Clinically Important Difference of the Quick Disabilities of the Arm Shoulder and Hand (QDASH) for Distal Upper Extremity Conditions" (2016). Physical Therapy Faculty Publications. 53.

https://uknowledge.uky.edu/rehabsci_facpub/53

This Article is brought to you for free and open access by the Physical Therapy at UKnowledge. It has been accepted for inclusion in Physical Therapy Faculty Publications by an authorized administrator of UKnowledge. For more information, please contact UKnowledge@lsv.uky.edu. 
Specificity of the Minimal Clinically Important Difference of the Quick Disabilities of the Arm Shoulder and Hand (QDASH) for Distal Upper Extremity Conditions

Digital Object Identifier (DOI)

http://dx.doi.org/10.1016/j.jht.2015.09.003

Notes/Citation Information

Published in Journal of Hand Therapy, v. 29, no. 1, p. 81-88.

Published by Elsevier Inc. on behalf of Hanley \& Belfus, an imprint of Elsevier Inc.

(c) 2016. This manuscript version is made available under the CC-BY-NC-ND 4.0 license

http://creativecommons.org/licenses/by-nc-nd/4.0/.

The document available for download is the authors' post-peer-review final draft of the article. 


\section{Published by Elsevier Inc. on behalf of Hanley \& Belfus, an imprint of Elsevier Inc.}

(C) 2016. This manuscript version is made available under the CC-BY-NC-ND 4.0 license http://creativecommons.org/licenses/by-ncnd/4.0/ 


\section{Accepted Manuscript}

Journal of

Hand Therapy

Specificity of the Minimal Clinically Important Difference of the Quick Disabilities of the Arm Shoulder and Hand (QDASH) for Distal Upper Extremity Conditions

Major(P) Enrique, V. Smith-Forbes, PhD, OTR/L, CHT, Dana, M. Howell, PhD, OTD, OTR/L, Jason Willoughby, MHS, OTR/L, CHT, Donald, G. Pitts, MS, OTR/L, CHT, Tim, L. Uhl, PT, ATC, PhD, FNATA

PII: $\quad$ S0894-1130(15)00166-0

DOI: $\quad$ 10.1016/j.jht.2015.09.003

Reference: HANTHE 931

To appear in: Journal of Hand Therapy

Received Date: 26 November 2014

Revised Date: 25 August 2015

Accepted Date: 21 September 2015

Please cite this article as: Smith-Forbes E,V, Howell D,M, Willoughby J, Pitts D,G, Uhl T,L, Specificity of the Minimal Clinically Important Difference of the Quick Disabilities of the Arm Shoulder and Hand (QDASH) for Distal Upper Extremity Conditions, Journal of Hand Therapy (2015), doi: 10.1016/ j.jht.2015.09.003.

This is a PDF file of an unedited manuscript that has been accepted for publication. As a service to our customers we are providing this early version of the manuscript. The manuscript will undergo copyediting, typesetting, and review of the resulting proof before it is published in its final form. Please note that during the production process errors may be discovered which could affect the content, and all legal disclaimers that apply to the journal pertain. 
Specificity of the Minimal Clinically Important Difference of the Quick Disabilities of the Arm Shoulder and Hand (QDASH) for Distal Upper Extremity Conditions

3

4

5

6

Authors:

Major(P) Smith-Forbes, Enrique, V., PhD, OTR/L, CHT ${ }^{a, *}$

Howell, Dana, M., PhD, OTD, OTR/L $L^{b c}$

Willoughby, Jason, MHS, OTR/L, CHT

Pitts, Donald, G., MS, OTR/L, CHT

Uhl, Tim, L., PT, ATC, PhD, FNATA ${ }^{b}$

${ }^{a}$ Graduate Medical Education, Fort Sam Houston Clinic, Building 1179, Room 1A43, 3100 Schofield Road, Fort Sam Houston, TX 78234, USA

${ }^{b}$ Department of Rehabilitation Sciences, College of Health Sciences, University of Kentucky, Lexington, KY, USA

${ }^{c}$ Department of Occupational Therapy, Eastern Kentucky University, Richmond, KY, USA

${ }^{d}$ Kentucky Hand \& Physical Therapy/ Drayer Physical Therapy Institute, Lexington, KY, USA

This study was presented in part at the $37^{\text {th }}$ American Society of Hand Therapy 2014 Meeting in

Boston, MA, and earned the 'The First Time Scientific Session Presenter Award.' This study

fulfilled in part the degree requirements for the first author. At the time of the study MAJ(P)

Smith-Forbes was affiliated with University of Kentucky.

* Corresponding author. Tel.: +1 832971 7757; fax: +1 8593236003.

3 E-mail address: enrique.smith-forbes@uky.edu; e_vsf12@hotmail.com (E.V.Smith-Forbes). 
ABSTRACT: Retrospective cohort design. The minimal clinically important difference (MCID) for the quick Disabilities of the Arm, Shoulder and Hand (QDASH) has been established using a pool of multiple conditions, and only exclusively for the shoulder. Understanding diagnosesspecific threshold change values can enhance the clinical decision-making process. Before and after QDASH scores for 406 participants with conditions of surgical distal radius fracture, nonsurgical lateral epicondylitis, and surgical carpal tunnel release were obtained. The external anchor administered at each fourth visit was a 15-point global rating of change scale. The testretest reliability of the QDASH was moderate for all diagnoses: intraclass correlation coefficient model 2,1, for surgical distal radius $=0.71$; non-surgical lateral epicondylitis $=0.69$; and surgical carpal tunnel $=0.69$. The minimum detectable change at the $90 \%$ confidence level was 25.28 ; 22.49; and 27.63 points respectively; and the MCID values were 25.8; 15.8 and 18.7, respectively. For these three distal upper extremity conditions, a QDASH MCID of 16-26 points could represent the estimate of change in score that is important to the patient and guide clinicians through the decision making process.

KEY WORDS: disability evaluation, musculoskeletal diseases, outcome assessment, psychometrics, rehabilitation, upper extremity

Level of Evidence: 2c 
1

2

3 standardized assessment that is perceived to be beneficial or harmful by the patient. ${ }^{1}$ The MCID

\section{INTRODUCTION}

The Minimal Clinically Important Difference (MCID) represents a change in score on a may be calculated for patients with upper extremity (UE) deficits using two common UE assessments, the quick Disabilities of the Arm, Shoulder and Hand (QDASH) ${ }^{2}$ and The Global Rating of Change (GROC). ${ }^{3}$ The MCID can be clinically used to interpret patient change scores to guide clinical decision-making.

The QDASH, a region specific outcome measure, is a shortened version of the Disabilities of the Arm Shoulder and Hand (DASH). ${ }^{4}$ Both instruments are widely used in rehabilitation., ${ }^{5,6}$ The GROC, a generic global change scale, allow patients to decide how much they have changed during recovery. The QDASH's MCID has been determined using the GROC to identify those patients who have improved and comparing them to those who have not improved with UE diagnoses. ${ }^{7}$ However, the results of these studies have generated a wide range of MCID (8-20), ${ }^{7-11}$ which represents $10-20 \%$ of the 100 -point scale and suggests the instrument may have poor responsiveness. One potential explanation for this variance may be because a single diagnosis was not used in most of the previous studies. ${ }^{7}$ The MCID may differ among diagnoses, and this may help explain the varying results in the literature. ${ }^{12}$ This is the primary rationale for examining MCID among separate diagnoses.

The QDASH’s psychometric and clinimetric properties have been investigated. Rasch analysis $^{13}$ and classical theory ${ }^{14-16}$ have been used to investigate the strength and weaknesses of the QDASH measures. A recent systematic review found the QDASH English version tool to perform well with strong positive evidence for reliability and validity (hypothesis testing) and moderate positive evidence for structural validity testing. Strong negative evidence was found 
24 for responsiveness due to lower correlations with global estimates of change. ${ }^{17}$

25 Multiple approaches have been used to calculate the responsiveness of these measures.

26 The MCID current and previous values become critical in assisting providers in making clinical

27 decisions. Several authors have suggested clinicians and researchers work with a range of MCID

28 values instead of a fixed value, ${ }^{18,19}$ another has questioned the validity of a single overall MCID. ${ }^{8}$

29 Distribution-based and anchor-based methods have been the two general approaches used to

30 interpret changes. The strategy for distribution-based approaches lies in identifying the Minimal

31 Detectable Change (MDC), which is the smallest change in score that can be distinguished

32 beyond random error. ${ }^{20}$ Distribution-based approaches do not give a good indication of the

33 importance of the observed change and therefore cannot provide the MCID. ${ }^{18}$ In contrast, with

34 anchor-based methods the choice of the anchor among other things will determine the precision

35 of the MCID.

36 Recent studies recommend the MCID be based primarily on anchor-based procedures, ${ }^{21}$

37 not be based on one study ${ }^{1}$ and should be higher than the MDC values (the typical boundary of

38 stable patients), ${ }^{20,21}$ and not be based on a single study. ${ }^{1}$ Nevertheless, there are limited studies

39 calculating the MCID through anchor-based approaches for the QDASH. ${ }^{7-10}$ Furthermore, it

40 seems the best option to determine MCID is to select a small range of threshold estimates from

41 the same sample and compare and interpret multiple reference standards. ${ }^{1,21,22}$ This approach has

42 been applied in a few studies on the DASH and QDASH. ${ }^{11,16}$ Some of the approaches to

43 calculate the MCID utilized in the literature are: $0.2 \mathrm{x}$ standard deviation at baseline, $0.5 \mathrm{x}$

44 standard deviation at baseline, and one standard error of measurement (test-retest), among many

45 others. $^{16}$

46 The main aim of this study was to use both anchor-based and distribution methods to 
triangulate on MCID values for the QDASH. We used a retrospective large sample of patients with UE musculoskeletal disorders who had undergone hand therapy. The objective was to determine condition specific thresholds for the MCID in order to enhance confidence in interpreting patient change scores for clinical decision-making.

\section{METHODS}

\section{Subjects}

This retrospective study population consisted of patients in a database seen at an outpatient UE orthopedic condition rehabilitation multi-center, over the last 4 years. There were approximately 5,000 patients in the existing database treated for multiple orthopedic conditions. All data in the database was de-identified and transferred to a data sheet for study purposes and then provided to the primary investigator (PI) for use by the database manager. The University of Kentucky’s Institutional Review Boards approved this exempt category study prior to data analysis.

\section{Inclusion and Exclusion Criteria}

Subjects age 18-89, were included if they were not missing QDASH scores at initial visit and visit 4, not missing last visit score determined per diagnoses at either visit 8 or visit 12, and not missing associated GROC scores for the QDASH. Diagnoses not totaling at least 100 records, based on the above criterion were excluded. Surgical distal radius fracture, non-surgical lateral epicondylitis, and carpal tunnel release were included as the three most common conditions treated by hand therapists at these facilities.

\section{Assessment}

The QDASH uses 11 items to measure the degree of difficulty in performing various physical activities due to a shoulder, arm, or hand problem. It utilizes a 5-point Likert scale for 
70

71

72

73

74

75

76

77

78

79

80

81

82

83

84

85

86

87

88

89

90

91

92

seven functional items and three symptom items. Ten of the 11 items need to be completed for the scores to be valid. The score is calculated on a 0-to-100 point scale. A higher score reflects greater disability. The 2 optional scales of the QDASH (work and sport/music) are not commonly collected in this clinical practice and therefore were not part of this study.

In contrast, the GROC scale ${ }^{23}$ asks that a person assess his or her current health status in relation to when they start their treatment and rate their level of change on a 15-point scale $(-7=$ a very great deal worse, $0=$ same, $+7=$ a very great deal better). ${ }^{24}$ Both instruments have been reported to be valid and reliable. ${ }^{2,25,26}$

\section{Procedure}

The database was reviewed to identify the most commonly treated diagnoses. It is known from review of the database that the typical number of visits for all diagnoses ranged from 8 to 12 visits. A screening process was used to identify that adequate scores were present at the time point of interest at initial, $4^{\text {th }}, 8^{\text {th }}$, and $12^{\text {th }}$ visit (Figure 1 ). In addition, the range of days treated was explored to determine a cutoff point for the last visit.

\section{Statistical Analysis}

Descriptive statistics

All statistical analyses were performed using Stata/ IC Version 13.1 (StataCorp LP, College Station, TX). Baseline characteristics per diagnoses between improved and not improved patients were determined for patient demographics of age, initial QDASH, and length of days in care using a $t$-test for parametric data and a Wilcoxon Mann-Whitney test for nonparametric data. A Chi-square test was used to calculate baseline gender differences (Table 1). ${ }^{9}$ Patients were sub-divided per diagnoses into two groups each, stable and improved, in order to analyze baseline characteristics. Stable patients were categorized from -2 to +3 . Improved patients were 
determined as reported scores on the GROC of $(\geq+4),{ }^{9}$ at visit 12 or visit eight for carpal tunnel release.

\section{Validity and Reliability}

1) We examined Convergent Validity to determine the correlation between the QDASH and the GROC using Pearson correlation coefficient (r). This was performed because the GROC was the reference standard, or external criteria by which we judged that a real patient improvement had occurred. We expected an at least a fair association $(\mathrm{r}>0.30)$ between their final QDASH score (visit eight or twelve), and their final GROC score (visit eight or twelve).

2) Test-retest reliability was calculated for the QDASH using an ANOVA (ICC2,2,1) using a group of stable patients on GROC $(-2$ to +2$) .{ }^{9}$ In order to assess reliability, the fourth visit of the QDASH was compared to the initial visit scores, as they were the earliest available repeated QDASH scores.

\section{Responsiveness}

Responsiveness was determined by distribution-based and anchor-based methods.

a) Distribution-based methods determine the ability to detect change in general, and are based on the statistical characteristics of the sample. We calculated the Standard Error of Measurement (SEM), which links the reliability of a measurement tool to the standard deviation of the population. This was obtained from an ANOVA using the entire population for the diagnosis. We calculated the Minimal Detectable Change (MDC), which represents the smallest change in score likely to reflect a true change, free from measurement error, $(\mathrm{MDC}=\mathrm{SEM} * \mathrm{Z}-$

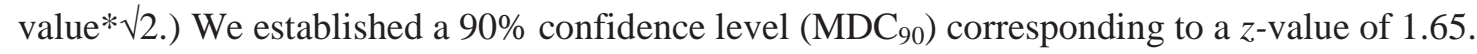
Meaning: If the patient has a change score greater or equal to the $\mathrm{MDC}_{90}$ threshold it is possible 
to state with $90 \%$ confidence that this change is real and not due to measurement error.

b) Anchor-based methods utilize an external patient criterion (an anchor) to determine if changes in outcome are clinically meaningful. Two approaches were used; the mean change and receiver-operating-characteristic (ROC) curve approaches. The GROC assessment was used as the external reference in evaluating responsiveness.

c) The Mean Change Approach: Was calculated as the mean change score in the different subgroups of patients who respectively reported themselves as not improved ( -7 to 0 ), minimally improved ( +1 to +3$)$, moderately improved $(+4$ to +5$)$ and large changes (+6 to +7$)$. We used changes in those minimally improved to triangulate the MCID values.

d) The ROC Curve Approach: We determined the optimal cutoff score and the area under the curve (AUC) considering the subjects improved with a GROC of +4 or greater.

A ROC curve plots sensitivity (y-axis) against 1 - specificity (x-axis). Following this rationale, sensitivity was calculated as the number of patients correctly identified as improved based on the cutoff value divided by all patients identified as having had a meaningful change (GROC +4 or greater), whereas specificity refers to the number of patients who were correctly identified as not improved based on the cutoff value divided by all patients who truly did not have a meaningful change (GROC, less than +4). The optimal cutoff was chosen as the point that jointly maximized sensitivity and specificity (was associated with the least amount of misclassification).

The AUC can be interpreted as the probability that a given diagnostic tool will correctly assign a patient to the appropriate diagnostic category. In general, AUC values between 0.7 and 0.8 are judged as acceptable, and an AUC value greater than 0.8 is considered to have good to excellent discrimination. ${ }^{27}$ The greater the AUC, the greater a measure's ability to distinguish patients who have improved from those who have not improved. In accordance with Turner et 
$139 \mathrm{al},{ }^{28}$ our ROC analysis will use the entire cohort, rather than just those subjects with ratings

140 adjacent to the dichotomization point to increase accuracy and obtain more reasonable estimates

141 of the MCID. We used the ICC test-retest from the product of our ANOVA that utilized a GROC

142 of $(-2$ to +2$) .^{9}$

143 To obtain CIs for the ROC-derived parameters, we drew 50 bootstrap samples and

144 calculated both the cutoff value and the AUC in each bootstrap replication. The mean of the 50

145 bootstrap AUC values was taken as the best estimate, with the 95\% CI calculated as 1.96 . SD

146 (as an estimate of the standard error) of the bootstrap values. ${ }^{1}$ This was done because the AUC

147 does not provide a CI, which in turn provides an estimate of how acceptable are our findings (.50

148 not good .70 acceptable, .80 good).

149 The MCID was set at the best triangulation of the results coming from both anchor-based

150 (mean change and the ROC curve) and distribution-based (the $\mathrm{MDC}_{90}$ threshold) methods. This

151 is considering that the MCID should be based primarily on anchor-based procedures ${ }^{21}$ and be

152 higher than the MDC value. In this regard, the MDC should be interpreted as another piece in the 153 puzzle toward establishing the MCID, by benchmarking it to the boundaries of error. ${ }^{11}$

154 According to Turner et al, ${ }^{20}$ "if the two anchor-based methods calculated on the same 155 population yield different MCID values, then the knowledge that one value is below the MDC

156 could aid in the decision to select the other.” In addition, the ROC-curve approach was preferred

157 as the first choice as it successfully addresses most limitations of the mean change

158 approach. ${ }^{1,21,28}$ Furthermore, our calculation of the 95\% CIs gave a useful indication of the sam-

159 pling variation. ${ }^{18}$

160 RESULTS

161 Descriptive Statistics and Validity of the Measures 
After excluding for missing data, 406 patients met inclusion criteria for three diagnoses;

163

164

165

166

168

169

170

171

172

173

174

175

176

177

178

179

180

\section{Responsiveness}

182 Distribution-based methods

183

184 shown in Table 3. surgical distal radius fracture $(\mathrm{n}=151)$, non-surgical lateral epicondylitis $(\mathrm{n}=137)$, and carpal tunnel release ( $\mathrm{n}=118)$. Most demographical data yielded no significant differences between improved and not improved groups with exception of lower initial QDASH scores for the improved group for surgical distal radius fracture, $P=.006$ and gender for carpal tunnel release, $P=.04$, see Table 1. Scores for the QDASH (initial and last visit), last visit GROC, as well as cutoff treatment sessions and duration of treatment days are presented in Table 2. Based on a previous study consisting of multiple diagnoses, with an average duration of 10 visits /22 days, ${ }^{11}$ a cutoff of 12 visits was chosen for surgical distal radius fracture and non-surgical lateral epicondylitis. A cutoff of 8 visits for carpal tunnel release occurred due to a shorter duration, see Table 2. Mean score changes for the QDASH questionnaire according to each GROC grade are

The correlation between GROC and the score changes of the QDASH was significant for all three diagnoses with a fair relationship for surgical distal radius fracture $(r=0.39, P<0.001)$ and for non-surgical lateral epicondylitis $(r=0.39, P<0.001)$, and a weak, but significant relationship for carpal tunnel release $(r=0.22, P=0.029$.) The test-retest reliability using a group of stable patients on GROC (-2 to +2), had moderate agreement for all three diagnoses surgical distal radius fracture: $\mathrm{ICC}_{2,1}=0.71$, (95\% CI: 0.51, 0.83)- non-surgical lateral epicondylitis: 0.69, (95\% CI: 0.56, 0.79)- and carpal tunnel release: 0.69, (95\% CI: 0.43, 0.84).

For the surgical distal radius fracture the SEM was 10.83 and the $\mathrm{MDC}_{90}$ corresponded to 25.28, for the non-surgical lateral epicondylitis the SEM was 9.63, and the $\mathrm{MDC}_{90}$ was 22.49; 
185 and for the carpal tunnel release the SEM was 11.84, and the MDC 90 was 27.63.

186 Anchor-based methods

187 The mean changes for the QDASH, per diagnoses, are reported in Table 3. In particular

188 those patients who were rated as having a small improvement (GROC, +1 to +3$)$ had a mean

189 change improvement for surgical distal radius fracture of 25.8 points (95\% CI: $14.4,35.6)$ for the

190 QDASH; for non-surgical lateral epicondylitis of 15.3 points (95\% CI: 11.4, 19.1); and for carpal

191 tunnel release of 18.7 points (95\% CI: 8.5,25.2). Splitting the data according to a presence of

192 moderate or larger improvement $(\geq+4)$ versus the remainder of the entire cohort, the AUC for

193 the QDASH for surgical distal radius fracture was 0.66 (95\% CI: 0.56, 0.77), (Figure 2); 0.64,

194 (95\% CI: 0.55, 0.73), (Figure 3); and for carpal tunnel release 0.66, (95\% CI: 0.55, 0.77), (Figure

195 4). The ROC-curve cutoff scores that best identified meaningful improvement in clinical status

196 (as measured by GROC values of +4 or greater) for surgical distal radius fracture 15.8 points

197 (95\% CI: -5.3, 36.9); for non-surgical lateral epicondylitis 15.8 points (95\% CI: 1.0, 30.6) points;

198 and for carpal tunnel release 13.3points $(-1.7,28.3)$ for the QDASH.

199 Surgical distal radius fracture triangulation

200 We took into account the following data (a) an $\mathrm{MDC}_{90}$ of 25.28 points for the QDASH,

201 (b) a mean change for small improvement of 25.8 points for the QDASH, and (c) an ROC cutoff

202 score that best identified meaningful improvement in clinical status of 15.8 points (sensitivity

203 86\%, specificity 37\%, correctly classified 74\%), for the QDASH. Analyzing the overall results

204 we had two competing anchor-based methods, the mean change $=25.8$ and the ROC $=15.8$.

205 Based on Turner et al, ${ }^{20}$ recommendations, the MCID $=25.8$, was selected since it was just right

206 over the $\mathrm{MDC}_{90}=25.28$ points.

207 Non-surgical lateral epicondylitis triangulation 
We took into account the following data (a) an MDC $_{90}=22.49$ points for the QDASH,

(b) a mean change for small improvement of 15.3 points for the QDASH, and (c) an ROC cutoff

210 score that best identified meaningful improvement in clinical status of 15.8 points (sensitivity

211 65\%, specificity 59\%, correctly classified 63\%) for the QDASH. Analyzing the overall results

212 our two anchor-based methods yielded similar results, the mean change $=15.3$ and the ROC $=$

213 15.8. However, both values were lower than the MDC $_{90}$ of 22.49 points. Therefore, we selected a

214 MCID $=15.8$ points from the AUC since it was the closest to the MDC 9 .

215

216

217

218

220

221

222

223

224

\section{Carpal tunnel release triangulation}

We took into account the following data: (a) an $\mathrm{MDC}_{90}$ of 27.63 points for the QDASH, (b) a mean change for small improvement of 18.7 points for the QDASH, and (c) an ROC cutoff score that best identified meaningful improvement in clinical status of 13.3 points (sensitivity 76\%, specificity 50\%, correctly classified 69\%) for the QDASH. Analyzing the overall results we had competing values of mean change $=18.7$, and an $\mathrm{ROC}=13.3$ points. However, again both values were lower than the MDC $_{90}$ of 27.63 points. Therefore, we selected a MCID $=18.7$ points from the mean change approach, since it was the closest to the $\mathrm{MDC}_{90}$.

\section{DISCUSSION}

In this era of evidence-based medicine, patients, clinicians and third-party payers demand to know the effectiveness of therapeutic interventions. This study contributes to the body of knowledge on the psychometric properties of the QDASH by examining the MCID for three distal upper extremity conditions: surgical distal radius fracture, non-surgical lateral epicondylitis, and carpal tunnel release.

In order to assess reliability, the fourth visit of the QDASH was compared to the initial visit scores, as they were the earliest available repeated QDASH scores. The average time from 
231 the initial to fourth QDASH visit were $9 \pm 3$ days for surgical distal radius fracture, $10 \pm 6$ days

232 for non-surgical lateral epicondylitis, and $11 \pm 7$ days for the carpal tunnel release. The test-retest

233 reliability for all three diagnoses ranged from 0.69 to 0.71 , indicating moderate agreement.

234 Mintken et al., found a higher reliability of 0.90 examining a cohort of shoulder patients. ${ }^{9}$

235 Although, in our study the average length of days between tests was 10 days, which may have

236 contributed to recall bias. In Mintken et al's., study the average length of follow-up time was

237 even larger at 27 days.

238 This study used anchor-based and distribution-based methods to triangulate and assess

239 the MCID for the QDASH on three diagnoses: surgical distal radius fracture, non-surgical lateral

240 epicondylitis, and carpal tunnel release. During the triangulation of our results we considered

241 that the MCID should be based primarily on anchor-based procedures, and in the first instance on

242 the ROC curve, ${ }^{11,21,29}$ and if possible, to be higher than the MDC value. ${ }^{30}$

243 Regarding the distribution-based approach, in our sample the $\mathrm{MDC}_{90}$ for all three

244 diagnoses was larger than the ROC calculated values. This is not uncommon ${ }^{9,31}$ as distributional

245 approaches are complicated by competing suggestions for the “'beyond error”' thresholds (e.g., 1,

246 1.96, or $2.77 \mathrm{SEM}) .{ }^{32,33}$ Some authors have recommended a more reliable method to estimate the

247 MDC is to calculate 0.5 of the SD or 1 SEM. $^{20}$ Applying this method, all our MDC9o's would fall

248 below the ROC calculated values. For the three diagnoses, the $\mathrm{MDC}_{90}$ values obtained were

249 above 20 points, and were larger than what is commonly reported in the literature. One reason

250 may be due to the retrospective nature of the data as higher quality control could have been

251 provided in a prospective study design. Nevertheless, one strength of this study was that all data

252 were collected on patients being treated in the course of normal hand therapy. The retrospective

253 nature is a limitation, but it is more indicative of a real and typical clinical result as this is exactly 
254 what it is. Patients may or may not participate in a study due to time limitation. However, these 255 data were collected as a standard operation procedure and were extracted after the fact. This data 256 has strong external validity due to the manner in which it was originally collected. The MCID measures important change because it uses a patient generated anchor for comparison. In contrast, the MDC measures statistical distribution of margins of error. ${ }^{20}$ Following Turner et al's recommendation, the $\mathrm{MDC}_{90}$ was regarded as a benchmark to establish margins of error for the MCID, and in our sample it represented the higher bound. ${ }^{11,20}$ Regarding the anchor-based method, the first concern about the appropriateness of the cutoff values is the selection of the anchor. We used a 15 -point anchor $(-7=$ a very great deal worse, $0=$ same, $+7=$ a very great deal better) and considered patients +4 to +7 as significantly improved and others as not significantly improved, to utilize the entire cohort. ${ }^{28}$ There is no agreement in the literature on what type of GROC's to use, which groups to include in the analysis, or the level at which to dichotomize. ${ }^{11,28}$ Furthermore, different standards have been used to determine and select the 267 cutoff values for the QDASH. ${ }^{2,9-11}$ In addition, it is difficult to make any direct comparisons to 268 MCID’s due to the methods employed including the choice of anchor, decision rules and types of 269 calculation procedures. ${ }^{11,20}$ In our sample, we found the ROC yielded values that were smaller 270 than the mean change approach within each category of small, moderate, and large changes, with 271 one exception (small changes for non-surgical lateral epicondylitis) which is similar to the MCID 272 review findings by Turner et al. ${ }^{20}$ See Table 3.

273 We found the ROC values to fall within previously established MCID estimates for the 274 QDASH ranging from 8 to 20 points. ${ }^{11}$ In particular, two of our ROC values of 15.8 points for 275 the surgical distal radius fracture and non-surgical lateral epicondylitis were similar to recent 276 estimates by the Franchignoni group at 15.91 points. ${ }^{11}$ However, based on the recommended 
methods of triangulation in the literature, the ROC value was only selected for non-surgical lateral epicondylitis. After triangulation, only one of our MCID values (post-surgical distal radius fracture, 25.8 points) fell outside the upper limit of 20 points reported in the literature. Overall, one benefit of this sample is that it is one of the largest groups of patients to examine the responsiveness of the QDASH.

In a recent review measuring clinical outcomes for distal radius fractures, pain and function were regarded as the primary domains out of seven core areas of recommendations. ${ }^{34}$ Considering this, in our study one explanation for a larger MCID for the two post-surgical diagnoses, may be the perceived initial pain and edema restrictions from the surgical intervention. Patients can be limited by the anticipation of pain and expectations of decreased function following surgery. ${ }^{35}$ Therefore, patients may perceive the need to regain greater ROM and decrease pain before they can report a minimal improvement in their status. This reasoning is supported by another study that examined patient satisfaction with outcomes after surgical distal radius fractures. ${ }^{36}$ That study concluded patients need to regain greater wrist range of motion than what is necessary to perform activities of daily living, to be satisfied with treatment outcomes. $^{36}$

\section{Limitations}

Patient baseline status and patient demographics can significantly affect MCID scores. ${ }^{37}$ In our study there were significant baseline QDASH differences for surgical distal radius fracture, $P=.006$; and gender for carpal tunnel release, $P=.04$. Therefore, the MCID should be interpreted with caution. It is important to note the MCID will fluctuate based on what is important to the patient, as it is not a fixed value, ${ }^{37}$ and will vary based on the method chosen to determine the MCID, as well as the type of population. ${ }^{29}$ For this reason, the results of this study 
300

301

302

303

304

305

306

307

308

309

310

311

313

can only be generalized to those groups of patients and individuals with similar characteristics to this sample. ${ }^{31}$ In addition, the use of the GROC may have introduced recall bias and the use of a retrospective sample, without pre-existing controls, may explain the large $\mathrm{MDC}_{90}$ obtained for each diagnosis as above indicated.

\section{CONCLUSION}

This study proposes the specific MCID values for the surgical distal radius fracture, nonsurgical lateral epicondylitis, and carpal tunnel release diagnoses, based on a comprehensive triangulation of anchor-based and distribution-based approaches. ${ }^{11}$ Based on triangulation rules, ${ }^{1,16,20,21}$ we selected MCID values of 25.8 points for surgical distal radius fracture, 15.8 points for non-surgical lateral epicondylitis, and 18.7 points for carpal tunnel release. The respective $\mathrm{MDC}_{90}$ values can serve as margins of error $^{20}$ for surgical distal radius fracture (25.28), non-surgical lateral epicondylitis (22.49) and carpal tunnel release (27.63) points for the QDASH. We agree with other studies noting a need of the standardization of the MCID methodology. ${ }^{11,20,29}$

\section{Clinical Implications}

Clinicians can use these MCID scores for the surgical distal radius fracture, non-surgical lateral epicondylitis and carpal tunnel release to understand how much change represents a meaningful change to a patient with these specific diagnoses. Previously reported QDASH MCID values ranged from 8- 20 points. ${ }^{7-11}$ The results from this study indicate a MCID range of 16 to 26 points represents the minimal clinical change meaningful to patients presenting with three specific elbow and wrist conditions. Specifically, post-surgical distal radius fracture patients may need to have a larger improvement (25.8 points) than previously reported using a pool of conditions (up to 20 points). These diagnoses specific MCID’s can help guide decision- 
323 making during the course of treatment. The selected MCID’s serve as a gauge on how much

324 change a patient may need to undergo to experience a true change during the course of treatment,

325 while the MDC90's serve as error margins to the MCID’s.

326 


\section{References}

1. Terwee CB, Roorda LD, Dekker J, et al. Mind the MIC: large variation among populations and methods. Journal of clinical epidemiology. May 2010;63(5):524-534.

2. Beaton DE, Wright JG, Katz JN. Development of the QuickDASH: comparison of three item-reduction approaches. The Journal of bone and joint surgery. American volume. May 2005;87(5):1038-1046.

3. Kamper SJ, Maher CG, Mackay G. Global rating of change scales: a review of strengths and weaknesses and considerations for design. The Journal of manual \& manipulative therapy. 2009;17(3):163-170.

4. Hudak PL, Amadio PC, Bombardier C. Development of an upper extremity outcome measure: the DASH (disabilities of the arm, shoulder and hand) [corrected]. The Upper Extremity Collaborative Group (UECG). American journal of industrial medicine. Jun 1996;29(6):602-608.

5. Bot AG, Becker SJ, van Dijk CN, Ring D, Vranceanu AM. Abbreviated Psychologic Questionnaires Are Valid in Patients With Hand Conditions. Clinical orthopaedics and related research. Aug 32013.

6. Roy JS, MacDermid JC, Woodhouse LJ. Measuring shoulder function: a systematic review of four questionnaires. Arthritis and rheumatism. May 15 2009;61(5):623632.

7. Polson K, Reid D, McNair PJ, Larmer P. Responsiveness, minimal importance difference and minimal detectable change scores of the shortened disability arm shoulder hand (QuickDASH) questionnaire. Manual therapy. Aug 2010;15(4):404407.

8. Kennedy CA, Beaton DE, Solway S, McConnell S, Bombardier C. The DASH and QuickDASH Outcome Measure User's Manual. 3rd ed. Toronto, Ontario, Canada: Institute for Work and Health; 2011.

9. Mintken PE, Glynn P, Cleland JA. Psychometric properties of the shortened disabilities of the Arm, Shoulder, and Hand Questionnaire (QuickDASH) and Numeric Pain Rating Scale in patients with shoulder pain. Journal of shoulder and elbow surgery / American Shoulder and Elbow Surgeons ... [et al.]. Nov-Dec 2009;18(6):920-926.

10. Sorensen AA, Howard D, Tan WH, Ketchersid J, Calfee RP. Minimal clinically important differences of 3 patient-rated outcomes instruments. The Journal of hand surgery. Apr 2013;38(4):641-649.

11. Franchignoni F, Vercelli S, Giordano A, Sartorio F, Bravini E, Ferriero G. Minimal Clinically Important Difference of the Disabilities of the Arm, Shoulder and Hand Outcome Measure (DASH) and Its Shortened Version (QuickDASH). The Journal of orthopaedic and sports physical therapy. Jan 2014;44(1):30-39.

12. Van Vliet MM, Maradey JA, Homa KA, Kerrigan CL. The usefulness of patientreported measures for clinical practice. Plastic and reconstructive surgery. Jul 2013;132(1):105-112.

13. Franchignoni F, Ferriero G, Giordano A, Sartorio F, Vercelli S, Brigatti E. Psychometric properties of QuickDASH - A classical test theory and Rasch analysis study. Manual therapy. 2011;16(2):177-182. 
372

373

374

375

376

377

378

379

380

381

382

383

384

385

386

387

388

389

390

391

392

393

394

395

396

397

398

399

400

401

402

403

404

405

406

407

408

409

410

411

412

413

414

415

416
14. Angst F, Schwyzer HK, Aeschlimann A, Simmen BR, Goldhahn J. Measures of adult shoulder function: Disabilities of the Arm, Shoulder, and Hand Questionnaire (DASH) and its short version (QuickDASH), Shoulder Pain and Disability Index (SPADI), American Shoulder and Elbow Surgeons (ASES) Society standardized shoulder assessment form, Constant (Murley) Score (CS), Simple Shoulder Test (SST), Oxford Shoulder Score (OSS), Shoulder Disability Questionnaire (SDQ), and Western Ontario Shoulder Instability Index (WOSI). Arthritis care \& research. Nov 2011;63 Suppl 11:S174-188.

15. Beaton DE, Katz JN, Fossel AH, Wright JG, Tarasuk V, Bombardier C. Measuring the whole or the parts? Validity, reliability, and responsiveness of the Disabilities of the Arm, Shoulder and Hand outcome measure in different regions of the upper extremity. Journal of hand therapy: official journal of the American Society of Hand Therapists. Apr-Jun 2001;14(2):128-146.

16. Beaton DE, van Eerd D, Smith $P$, et al. Minimal change is sensitive, less specific to recovery: a diagnostic testing approach to interpretability. Journal of clinical epidemiology. May 2011;64(5):487-496.

17. Kennedy CA, Beaton DE, Smith $P$, et al. Measurement properties of the QuickDASH (Disabilities of the Arm, Shoulder and Hand) outcome measure and cross-cultural adaptations of the QuickDASH: a systematic review. Quality of life research : an international journal of quality of life aspects of treatment, care and rehabilitation. Mar 122013.

18. de Vet HC, Terluin B, Knol DL, et al. Three ways to quantify uncertainty in individually applied "minimally important change" values. Journal of clinical epidemiology. Jan 2010;63(1):37-45.

19. de Vet HC, Terwee CB, Ostelo RW, Beckerman H, Knol DL, Bouter LM. Minimal changes in health status questionnaires: distinction between minimally detectable change and minimally important change. Health and quality of life outcomes. 2006;4:54-54.

20. Turner D, Schunemann HJ, Griffith LE, et al. The minimal detectable change cannot reliably replace the minimal important difference. Journal of clinical epidemiology. Jan 2010;63(1):28-36.

21. Revicki D, Hays RD, Cella D, Sloan J. Recommended methods for determining responsiveness and minimally important differences for patient-reported outcomes. Journal of clinical epidemiology. Feb 2008;61(2):102-109.

22. Stratford PW, Riddle DL. When Minimal Detectable Change Exceeds a Diagnostic Test-Based Threshold Change Value for an Outcome Measure: Resolving the Conflict. Physical therapy. 2012;92(10):1338-1347.

23. Jaeschke R, Singer J, Guyatt GH. Measurement of health status. Ascertaining the minimal clinically important difference. Controlled clinical trials. Dec 1989;10(4):407-415.

24. Norman GR, Stratford P, Regehr G. Methodological problems in the retrospective computation of responsiveness to change: the lesson of Cronbach. Journal of clinical epidemiology. Aug 1997;50(8):869-879.

25. Gabel CP, Michener LA, Melloh M, Burkett B. Modification of the upper limb functional index to a three-point response improves clinimetric properties. Journal 
of hand therapy: official journal of the American Society of Hand Therapists. Jan-Mar 2010;23(1):41-51; quiz 52.

26. Wu A, Edgar DW, Wood FM. The QuickDASH is an appropriate tool for measuring the quality of recovery after upper limb burn injury. Burns : journal of the International Society for Burn Injuries. Nov 2007;33(7):843-849.

27. Wright AA, Cook CE, Baxter GD, Dockerty JD, Abbott JH. A comparison of 3 methodological approaches to defining major clinically important improvement of 4 performance measures in patients with hip osteoarthritis. The Journal of orthopaedic and sports physical therapy. May 2011;41(5):319-327.

28. Turner D, Schunemann HJ, Griffith LE, et al. Using the entire cohort in the receiver operating characteristic analysis maximizes precision of the minimal important difference. Journal of clinical epidemiology. Apr 2009;62(4):374-379.

29. Wright A, Hannon J, Hegedus EJ, Kavchak AE. Clinimetrics corner: a closer look at the minimal clinically important difference (MCID). The Journal of manual \& manipulative therapy. Aug 2012;20(3):160-166.

30. Dawson J, Doll H, Boller I, et al. Comparative responsiveness and minimal change for the Oxford Elbow Score following surgery. Quality of life research : an international journal of quality of life aspects of treatment, care and rehabilitation. Dec 2008;17(10):1257-1267.

31. Michener LA, McClure PW, Sennett BJ. American Shoulder and Elbow Surgeons Standardized Shoulder Assessment Form, patient self-report section: reliability, validity, and responsiveness. Journal of shoulder and elbow surgery / American Shoulder and Elbow Surgeons ... [et al.]. Nov-Dec 2002;11(6):587-594.

32. Bruynesteyn $\mathrm{K}$, van der Heijde D, Boers M, et al. Minimal clinically important difference in radiological progression of joint damage over 1 year in rheumatoid arthritis: preliminary results of a validation study with clinical experts. The Journal of rheumatology. Apr 2001;28(4):904-910.

33. Beaton DE, Boers M, Wells GA. Many faces of the minimal clinically important difference (MCID): a literature review and directions for future research. Current opinion in rheumatology. Mar 2002;14(2):109-114.

34. Goldhahn J, Beaton D, Ladd A, Macdermid J, Hoang-Kim A. Recommendation for measuring clinical outcome in distal radius fractures: a core set of domains for standardized reporting in clinical practice and research. Archives of orthopaedic and trauma surgery. 2014;134(2):197-205.

35. O'Brien L, Presnell S. Patient experience of distraction splinting for complex finger fracture dislocations. Journal of hand therapy: official journal of the American Society of Hand Therapists. Jul-Sep 2010;23(3):249-249; quiz 260.

36. Chung KC, Haas A. Relationship between patient satisfaction and objective functional outcome after surgical treatment for distal radius fractures. Journal of hand therapy : official journal of the American Society of Hand Therapists. Oct-Dec 2009;22(4):302-307; quiz 308.

37. Wang YC, Hart DL, Stratford PW, Mioduski JE. Baseline dependency of minimal clinically important improvement. Physical therapy. May 2011;91(5):675-688. 
Table 1

Baseline Statistics for improved patients and the not improved (scores represent means and standard deviations unless otherwise indicated)

\begin{tabular}{|c|c|c|c|c|c|c|c|c|c|}
\hline \multirow[t]{3}{*}{ Descriptor } & Surgical & Distal & & Nonsurgical & Lateral & & Carpal & & \\
\hline & Radius & Fracture & & Epicondylitis & & & Tunnel & Release & \\
\hline & IP $(n=114)$ & $\mathrm{NP}(\mathrm{n}=37)$ & $P$ & IP $(n=69)$ & NP $(n=68)$ & $P$ & IP $(n=84)$ & $N P(n=34)$ & $P$ \\
\hline Age & $56(14.1)$ & $52(12.6)$ & $.16^{\mathrm{a}}$ & $47(9.2)$ & $46(8.0)$ & $.47^{\mathrm{C}}$ & $53(12.0)$ & $53(11.9)$ & $.93^{\mathrm{c}}$ \\
\hline Gender, (\% male) & $31(27 \%)$ & $9(24 \%)$ & $.73^{\mathrm{b}}$ & $35(51 \%)$ & $31(46 \%)$ & $.55^{\mathrm{b}}$ & $23(27 \%)$ & $16(47 \%)$ & $.04^{\mathrm{b}}$ \\
\hline Initial QDASH & $60(19.8)$ & $70(22.3)$ & $.006^{\mathrm{c}}$ & $39(17.8)$ & $43(19.8)$ & $.23^{\mathrm{c}}$ & $56(23.6)$ & $55(22.7)$ & $.93^{\mathrm{c}}$ \\
\hline $\begin{array}{l}\text { Duration of treatment } \\
\text { (days of care) }\end{array}$ & $35(12.3)$ & $35(13.4)$ & $.73^{\mathrm{a}}$ & $41(12.6)$ & $38(10.1)$ & $.22^{\mathrm{a}}$ & $26(10.0)$ & $26(10.1)$ & $.77^{\mathrm{a}}$ \\
\hline
\end{tabular}

IP: Improved Patients; NP: Not-improved Patients; P: Significance a: Wilcoxon (Mann Whitney-U); b: Chi-square tests; c: t-test

QDASH: The Quick Disabilities of The Arm Shoulder and Hand 
Table 2

Scores of the QDASH and GROC

\begin{tabular}{lccc}
\hline Descriptor & $\begin{array}{c}\text { Surgical Distal } \\
\text { Radius Fracture }\end{array}$ & $\begin{array}{c}\text { Nonsurgical } \\
\text { Lateral Epicondylitis }\end{array}$ & $\begin{array}{c}\text { Surgical Carpal } \\
\text { Tunnel Syndrome }\end{array}$ \\
\hline Initial QDASH & $63 \pm 20.7$ & $41 \pm 18.8$ & $56 \pm 23.3$ \\
Last visit QDASH & $29 \pm 20.5$ & $24 \pm 15.6$ & $30 \pm 17.6$ \\
Last visit GROC & $3.4 \pm 2.0$ & $3.4 \pm 2.1$ & $4.8 \pm 1.7$ \\
Cutoff treatment sessions & 12 & 12 & 8 \\
Duration of treatment, d* & $35 \pm 13(21-97)$ & $39 \pm 11(24-92)$ & $25 \pm 9(14-56)$ \\
\hline $\mathrm{d}^{*}$ : Days of care, values are mean \pm SD (range). & & \\
QDASH: The Quick Disabilities of The Arm Shoulder and Hand. & \\
GROC: Global Rate of Change Scale.
\end{tabular}


Table 3

Mean score changes for the QDASH questionnaire according to each GROC scale grade

\begin{tabular}{ccccccc}
\hline & $\begin{array}{c}\text { Surgical Distal } \\
\text { Radius Fracture }\end{array}$ & & $\begin{array}{c}\text { Nonsurgical } \\
\text { Lateral Epicondylitis }\end{array}$ & $\begin{array}{c}\text { Surgical Carpal } \\
\text { Tunnel Syndrome }\end{array}$ \\
\hline & $\mathrm{n}(\%)$ & QDASH & $\mathrm{n}(\%)$ & QDASH & $\mathrm{n}(\%)$ & QDASH \\
\hline 0 or less & $4(3 \%)$ & 9.7 & $11(8 \%)$ & 2.6 & $7(6 \%)$ & 15.9 \\
+1 to +3 & $33(22 \%)$ & 25.8 & $57(42 \%)$ & 15.3 & $27(23 \%)$ & 18.7 \\
+4 to +5 & $54(36 \%)$ & 29.6 & $52(38 \%)$ & 17.6 & $57(48 \%)$ & 26.6 \\
+6 to +7 & $60(39 \%)$ & 44.3 & $17(12 \%)$ & 33.5 & $27(23 \%)$ & 34.3 \\
\hline
\end{tabular}

QDASH: The Quick Disabilities of The Arm Shoulder and Hand. GROC: Global Rate of Change Scale. 
Figure 1

Flow of charts meeting inclusion criteria

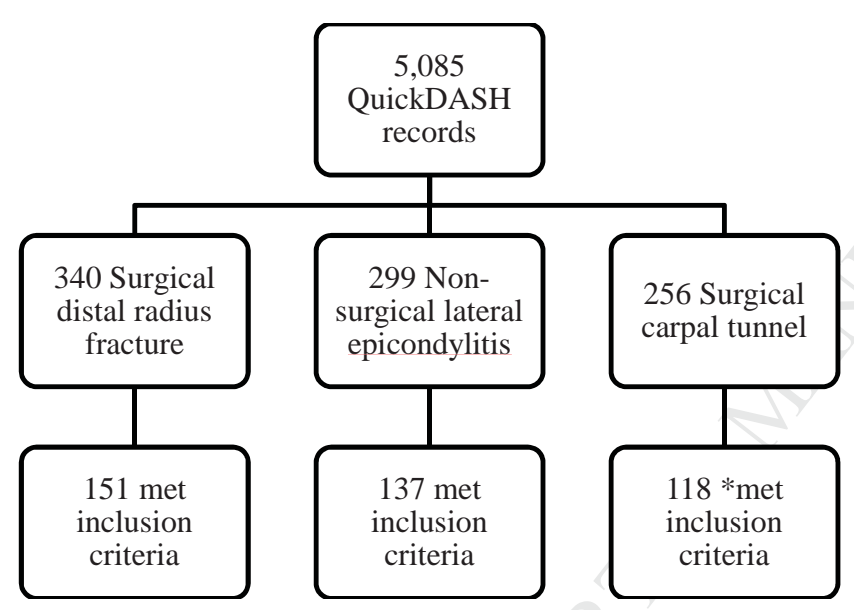

Inclusion criteria: have values for QDASH initial, visits 4,8 and 12, and GROC visit 12.

*= last visit for QDASH and GROC is visit 8 instead of 12 . 
Figure 2

QDASH Area Under The Curve (AUC) for surgical distal radius fracture.

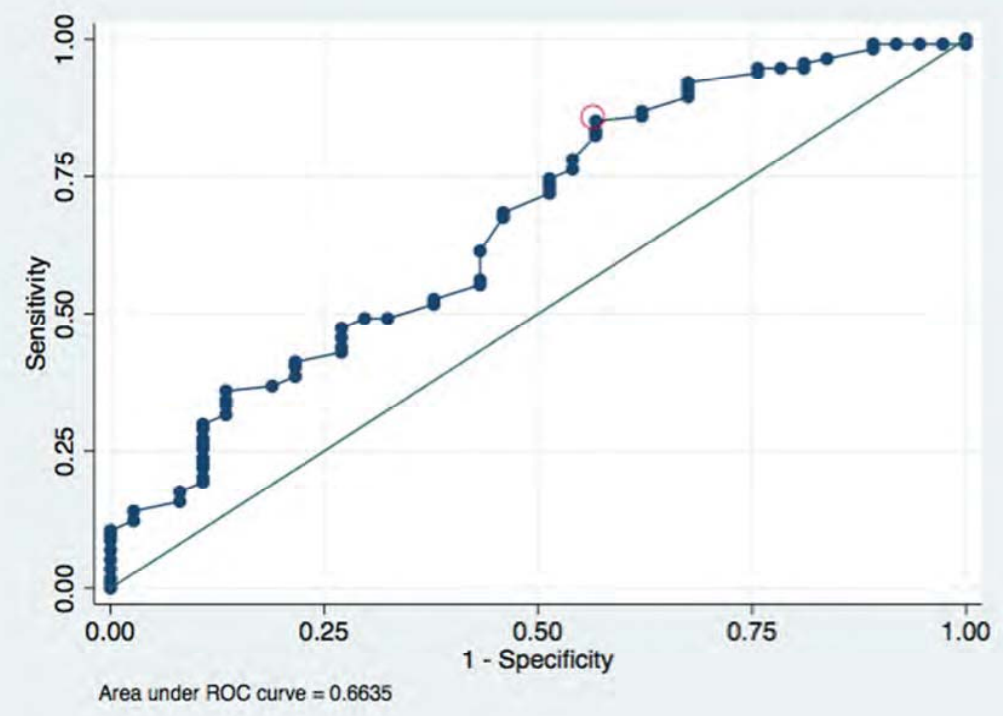


Figure 3

QDASH Area Under The Curve (AUC) for nonsurgical lateral epicondylitis.

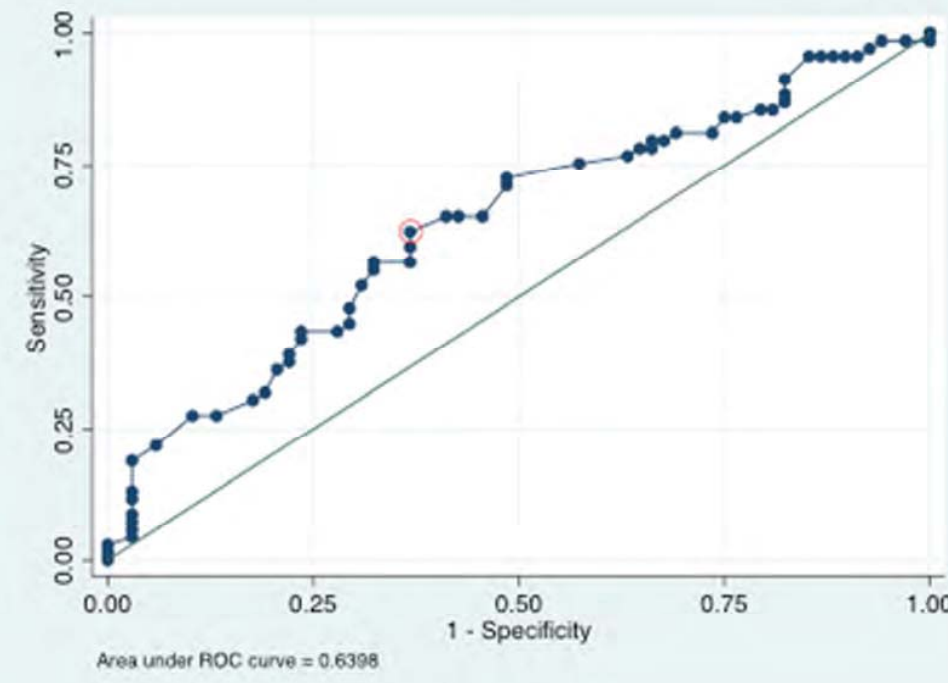


Figure 4

QDASH Area Under The Curve (AUC) for surgical carpal tunnel syndrome.

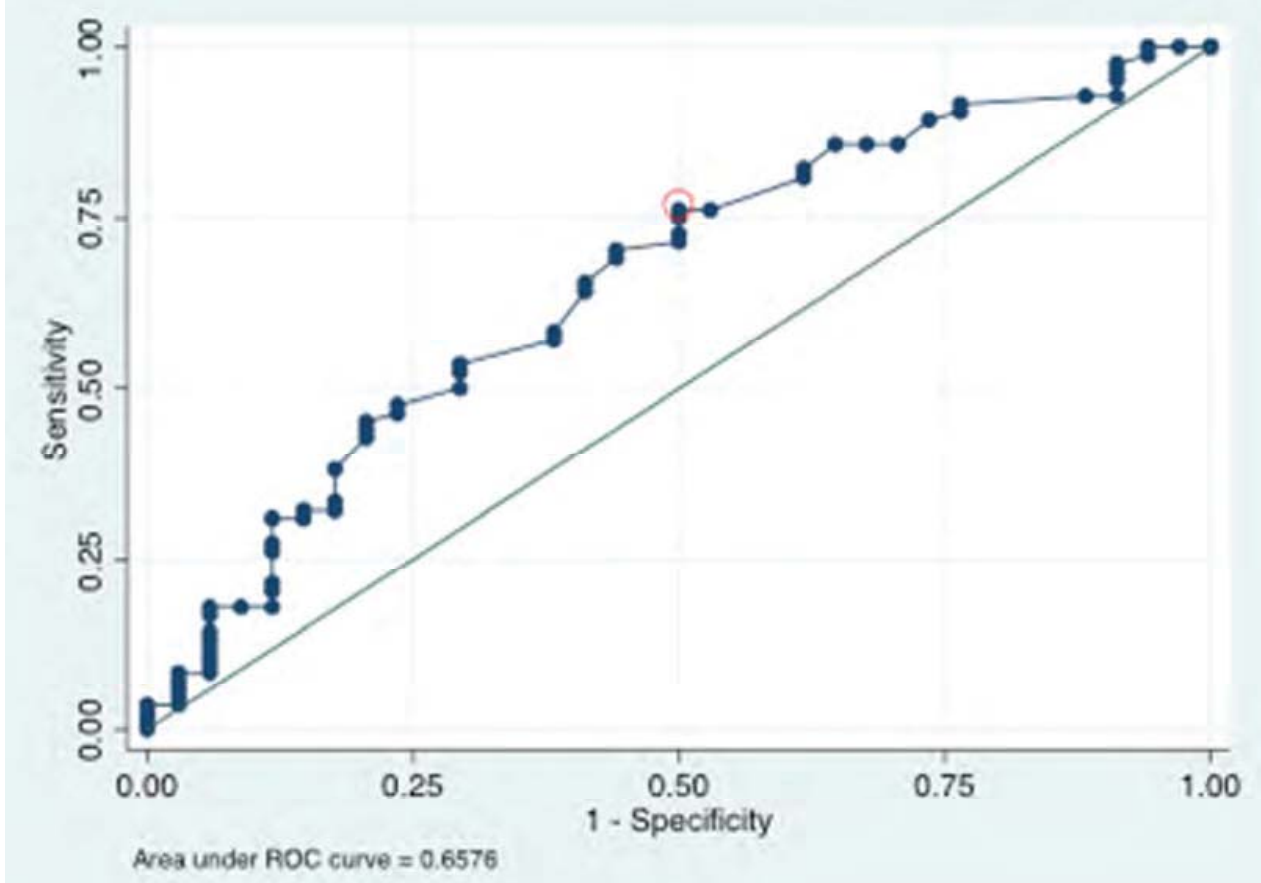


Highlights

- 406 participants, three diagnoses were assessed using triangulation methods

- For surgical distal radius fracture the $\mathrm{MCID}=25.8, \mathrm{MDC}_{90}=25.28$, and $\mathrm{AUC}=.66$

- For non-surgical lateral epicondylitis the $\mathrm{MCID}=15.8, \mathrm{MDC}_{90}=22.49$, and $\mathrm{AUC}=.64$

- For carpal tunnel release the MCID=18.7, $\mathrm{MDC}_{90}=27.63$, and $\mathrm{AUC}=.66$ 